Bull. Korean Math. Soc. 50 (2013), No. 2, pp. 441-444

http://dx.doi.org/10.4134/BKMS.2013.50.2.441

\title{
ON NULL CUT POINTS IN TWO-DIMENSIONAL SPACETIMES
}

\author{
Do-Hyung Kim and Byung HaK Kim
}

\begin{abstract}
It is shown that any two-dimensional globally hyperbolic spacetime with noncompact Cauchy surfaces has no null cut points.
\end{abstract}

\section{Introduction}

Conjugate point and cut point, defined in terms of Jacobi field along a geodesic and Riemannian distance, play important roles in global analysis of Riemannian manifold. Likewise, in Lorentzian geometry, they play essential roles in singularity theory of spacetimes. In connection with singularity theory, causality theory developed by Penrose [6], is an essential tool for global analysis of spacetimes. Lorentzian cut point, defined in terms of Lorentzian distance function, has a close relation to conjugate points and it is well-known that there is no null conjugate points in two-dimensional spacetimes ([1], [4], [5], [6]).

In contrast to this, there exists a two-dimensional spacetime in which every null geodesic has cut points which can be easily seen in two-dimensional Einstein static universe. In two-dimensional Einstein static universe, we can see that the spacetime has compact Cauchy surfaces.

In this paper, we show that if a two-dimensional Lorentzian manifold has noncompact Cauchy surfaces, then there exist no cut points along null geodesics by use of topological property of its Cauchy surface.

\section{Preliminaries}

In this section, we assume that $M$ is an arbitrary dimensional globally hyperbolic Lorentzian manifold with a noncompact Cauchy surface $\Sigma$. By the work of Bernal and Sánchez ([2] and [3]), we can assume that $\Sigma$ is a smooth, spacelike hypersurface. If there exists a future-directed timelike curve from $x$ to $y$, then we write $x \ll y$ and we say that $y$ lies in the chronological future

Received September 20, 2011.

2010 Mathematics Subject Classification. Primary 53B30, 53C50.

Key words and phrases. Lorentzian geometry, general relativity, causality, Cauchy surface, space-time, global hyperbolicity, null cut point.

The second author was supported by a grant from the Kyung Hee University in 2011(KHU20110480). 
of $x$ or $x$ lies in the chronological past of $y$. We define a subset $S \subset M$ to be achronal if no two points in $S$ are chronologically related. If there exists a future-directed causal curve from $x$ to $y$, then we write $x \leq y$ and we say that $y$ lies in the causal future of $x$ or $x$ lies in the causal past of $y$. We define $I^{+}(x)\left(I^{-}(x)\right.$, respectively) to be the set of all chronological future (past, respectively) of $x$ and $J^{+}(x)\left(J^{-}(x)\right.$, respectively) to be the set of all causal future (past, respectively) of $x$.

The relation " $x \leq y$ but not $x \ll y$ " is written $x \rightarrow y$ and is termed horismos. It is known that if $x \rightarrow y$, then any causal curve from $x$ to $y$ is a null geodesic without conjugate points except at most its endpoints (See Proposition 46 and Theorem 51 of Chapter 10 in [5]) and this is the main property which we use in this paper.

Definition. Let $S$ be an achronal subset of $M$. The edge of $S$ is the set of points $x$ such that for every neighborhood $U$ of $x$, there are two points $y$ and $z$ in $U$ and two timelike curves in $U$ from $y$ to $z$, just one of which meets $S$.

If $\Sigma$ is a non-compact Cauchy surface, then $S_{p}=J^{-}(p) \cap \Sigma$ is compact for each $p \in J^{+}(\Sigma)$ and, by the definition of edge points, it is easy to see that edge $S$ is precisely the set of boundary points of $S_{p}$ in $\Sigma$. Furthermore, since $\Sigma$ is noncompact and $S_{p}$ is compact, the boundary of $S_{p}$ in $\Sigma$ is non-empty and thus edge $S_{p}$ is non-empty for any $p \in J^{+}(\Sigma)$.

Proposition 2.1. If $x \in e d g e S_{p}$, then we have $x \rightarrow p$ and thus there exists a future-directed null geodesic from $x$ to $p$ without conjugate points except at most its endpoints.

Proof. Since $S_{p}$ is compact, it is closed and thus it contains its boundary points in $\Sigma$, which is precisely edge $S_{p}$. Therefore $x \in S_{p}$ and we have $x \leq p$. If $x \ll p$, then there exists a neighborhood $U$ of $x$ such that $y \ll p$ for all $y \in U$ since the relation $\ll$ is open. This contradicts to the fact that $x \in e d g e S_{p}$, which is the boundary of $S_{p}$ in $\Sigma$. Thus, we have $x \leq p$ but not $x \ll p$.

Proposition 2.2. $S_{p}$ is connected.

Proof. Let $X$ be a non-vanishing past-directed timelike vector field on $M$ and $\Phi$ be its flow. If we let $x$ and $y$ be arbitrary points in $S_{p}$, then we have two causal curves $\gamma_{1}$ from $x$ to $p$ and $\gamma_{2}$ from $y$ to $p$. By projecting $\gamma_{1} \cup-\gamma_{2}$ into $\Sigma$ through $\Phi$, we get a curve from $x$ to $y$ in $S_{p}$.

We can define null cut points in terms of Lorentzian distance function $d$ which is defined as, for $p \leq q, d(p, q)=\sup \{L(\gamma) \mid \gamma$ is a causal curve from $p$ to $q\}$ and $d(p, q)=0$ if $p \not \leq q$.

Definition. Let $\gamma:[0, a) \rightarrow M$ be a future-directed null geodesic from $p=$ $\gamma(0)$. If we let $t_{0}=\sup \{t \in[0, a) \mid d(p, \gamma(t))=0\}$ and if $0<t_{0}<a$, then we say $\gamma\left(t_{0}\right)$ is the future null cut point of $p$ along $\gamma$. Past null cut points are defined dually. 
By the above definition, null cut points are characterized by the property that if $\gamma(a)$ is a cut point of $\gamma(0)$ along a future-directed null geodesic, then we have $\gamma(0) \ll \gamma(t)$ for each $t>a$. Also, we can see that if $x \rightarrow y$, then any causal curve from $x$ to $y$ is a null geodesic without cut points except at most its endpoints.

\section{Main result}

In this section, let $M$ be a two-dimensional spacetime with a noncompact Cauchy surface $\Sigma$. Since the only noncompact one-dimensional manifold is the set of real numbers, $\mathbb{R}$, we have $\Sigma=\mathbb{R}$ and by the famous result of Bernal and Sánchez [2], we can assume that $M$ is diffeomorphic to $\mathbb{R} \times \mathbb{R}$. So we can use a natural coordinate system of $M$ as $\mathbb{R} \times \mathbb{R}$ and it naturally induces a coordinate system on $\Sigma$ as $\mathbb{R}$.

Lemma 3.1. For a two-dimensional spacetime $M$ with a noncompact Cauchy surface $\Sigma$, if $S_{p}=J^{-}(p) \cap \Sigma$ for $p \in I^{+}(\Sigma)$, then edge $S_{p}$ is non-empty and has exactly two points.

Proof. Since $M$ is two-dimensional, and $\Sigma$ is noncompact, we have that $\Sigma$ is diffeomorphic to $\mathbb{R}$. Since $S_{p}$ is a connected and compact subset of $\Sigma=\mathbb{R}, S_{p}$ must be of the form $[\alpha, \beta]$, the closed and bounded interval in $\mathbb{R}$. Therefore edge $S_{p}=\{\alpha, \beta\}$ since $e d g e S_{p}$ is precisely the set of boundary points of $S_{p}$ in $\Sigma=\mathbb{R}$.

For our main purpose, by symmetry, we only need to show that for any $p \in M$ and any past-directed null geodesic $\eta$ from $p, p$ has no null cut points along $\eta$.

Theorem 3.1. Let $M$ be a two-dimensional spacetime with noncompact Cauchy surfaces and let $\eta$ be a past-directed null geodesic from $p \in M$. Then $p$ has no null cut points along $\eta$.

Proof. Bernal and Sánchez ([2], [3]) have shown that a globally hyperbolic spacetime $M$ has a smooth function $f: M \rightarrow \mathbb{R}$ such that $f$ increases along any future-directed causal curves and its level set $f^{-1}(a)$ forms a smooth, spacelike Cauchy surface $\Sigma_{a}$ for each $a \in \mathbb{R}$. For our purpose, if we let $t=-f$, then $t$ increases along any past-directed causal curves and we can parameterize $\eta$ by $t$.

Let $t(p)=0$ and assume that $p=\eta(0)$ has a cut point at $\eta\left(t_{0}\right)$ along $\eta$. If $\Sigma_{t_{0}}$ is a Cauchy surface through $\eta\left(t_{0}\right)$, choose another Cauchy surface $\Sigma_{t_{1}}$ in $I^{-}\left(\Sigma_{t_{0}}\right)$. Then, by the previous lemma, $S_{p}\left(t_{1}\right)=J^{-}(p) \cap \Sigma\left(t_{1}\right)$ has two edge points, say, $\left(t_{1}, \alpha\right)$ and $\left(t_{1}, \beta\right)$. By Proposition 2.1, we have two null geodesics $\eta_{1}$ from $p$ to $\left(t_{1}, \alpha\right)$ and $\eta_{2}$ from $p$ to $\left(t_{1}, \beta\right)$. Since $\left(t_{1}, \alpha\right)$ and $\left(t_{1}, \beta\right)$ are edge points, $\eta_{1}$ and $\eta_{2}$ have no null cut points. Since $M$ is two-dimensional, the only past-directed null directions are either $\eta_{1}^{\prime}(0)$ or $\eta_{2}^{\prime}(0)$. Therefore, without loss of generality, we can assume that the direction of $\eta^{\prime}(0)$ is the same with that of 
$\eta_{1}^{\prime}(0)$. However, by the uniqueness of geodesic, this implies that $\eta=\eta_{1}$, which is a contradiction since $\eta$ has a cut point and $\eta_{1}$ does not.

If a globally hyperbolic spacetime has a future-directed null geodesic $\eta$ such that $\eta(a)$ is a cut point of $\eta(0)$ along $\eta$, then there is a future-directed timelike geodesic $\gamma$ from $\eta(0)$ to $\eta(a+\epsilon)$. By considering the fact that timelike geodesic is a freely falling observer, the existence of null cut point implies that there exists a freely falling observer with non-zero rest mass, who emits a light signal and he gets the emitted light signal after a finite time interval. The main theorem of this paper states that if a two-dimensional spacetime has a noncompact Cauchy surface, then no observer can catch up with light.

\section{References}

[1] J. K. Beem, P. E. Ehrlich, and K. L. Easley, Global Lorentzian Geometry, 2nd edition, Dekker, New York, 1996.

[2] A. N. Bernal and M. Sánchez, On smooth Cauchy hypersurfaces and Geroch's splitting theorem, Comm. Math. Phys. 243 (2003), no. 3, 461-470.

[3] _ Smoothness of time functions and the metric splitting of globally hyperbolic spacetimes, Comm. Math. Phys. 257 (2005), no. 1, 43-50.

[4] E. Minguzzi and M. Sánchez, The causal hierarchy of spacetimes, arxiv:gr-qc/0609119.

[5] B. O'Neill, Semi-Riemannian Geometry with Applications to Relativity, Pure and Applied ser., Vol. 103, Academic Press, New York, 1983.

[6] R. Penrose, Techniques of Differential Topology in Relativity, Regional Conference Series in Applied Mathematics, Vol. 7, SIAM Publications, Philadelphia, 1972.

Do-HYung KIM

Department of Applied Mathematics

College of Advanced Science

DANKOOK UNIVERSITY

Chungnam 330-714, Korea

E-mail address: mathph@dankook.ac.kr

Byung HaK Kim

Department of Applied Mathematics

College of Applied Science

KYUNG HeE UNIVERSITY

YONGIN 446-701, KoREA

E-mail address: bhkim@khu.ac.kr 